\title{
Diabetes mellitus como factor de riesgo de la periimplantitis
}

\author{
Diabetes mellitus as a risk factor for periimplantitis
}

Liz Guevara-Callire ${ }^{1 a b}$

Britto Ebert Falcón-Guerrero ${ }^{1 \mathrm{ac}}$

Nadia Flores-Chipana ${ }^{1 \mathrm{~d}}$, Luz Mamani-Mamani ${ }^{1 \mathrm{~d}}$, Rocío Mamani-Alejos ${ }^{\text {1d }}$, Hermes Mamani-Perea ${ }^{1 \mathrm{~d}}$, Sofía Ramos-Arce $^{1 \mathrm{~d}}$, Daniela Taya-Venegas ${ }^{1 \mathrm{~d}}$, Susana Yunganina-Laura ${ }^{1 \mathrm{~d}}$

Correspondencia: artdent2000@hotmail.com

\section{Resumen}

La diabetes mellitus es una enfermedad crónica endocrina que se considera como un factor de riesgo importante para el desarrollo de las enfermedades periodontales y periimplantarias debido a su naturaleza inflamatoria. El Objetivo de esta revisión es la de argumentar qué evidencia científica existe acerca de la diabetes mellitus como factor de riesgo para el desarrollo de la periimplantitis. Se realizó una revisión bibliográfica de los últimos 10 años (enero 2011 a enero 2021) usando términos de búsqueda que incluyeron las palabras clave en inglés: (dental implants OR oral implants) AND (diabetes OR diabetic), en los motores de búsqueda: PubMed y Embase (electronic databases). Concluyendo en que a pesar que no existe una evidencia concluyente, se debe considerar que existe relación entre la presencia de la diabetes mellitus y la periimplantitis; en tal razón se debe considerar a esta enfermedad como un factor de riesgo para la periimplantitis, siendo aconsejable que el paciente diabético debe mantener siempre controlado su nivel de glucosa.

Palabras clave: diabetes mellitus, implante dental, implante, periimplantitis, mucositis

\begin{abstract}
Diabetes mellitus is a chronic endocrine disease that is considered an important risk factor for the development of periodontal and peri-implant diseases due to its inflammatory nature. The objective of this review is to argue what scientific evidence exist on diabetes mellitus as a risk factor for the development of peri-implantitis. A bibliographic review of the last 10 years (January 2011 to January 2021) was carried out using search terms that included the keywords in English: (dental implants or oral implants) and (diabetes or diabetic), in search engines: PubMed and Embase (electronic databases). Concluding that although there is no conclusive evidence, it must be considered that there is a relationship between the presence of diabetes mellitus and peri-implantitis. For this reason, this disease should be considered as a risk factor for periimplantitis, and it is advisable that diabetic patients should always keep their glucose level under control.
\end{abstract}

Keywords: diabetes mellitus, implante dental, implante, periimplantitis, mucositis

\footnotetext{
${ }^{1}$ Universidad Latinoamericana CIMA. Facultad de Odontología. Tacna, Perú

${ }^{\text {a }}$ Docente

${ }^{\mathrm{b}}$ Maestro en Odontoestomatología

'Doctor en Estomatología. Especialista en Periodoncia e Implantología

${ }^{\mathrm{d}}$ Estudiante
} 


\section{Introducción}

Identificar los factores de riesgo en los pacientes que reciben un implante dental es esencial para pronosticar la aparición de la enfermedad y proporcionar una intervención preventiva adecuada; existiendo varios factores y complicaciones que podrían conducir a la pérdida del implante dental, como la mala higiene bucal, antecedentes de periodontitis previa a la instalación del implante y el hábito del tabaco; además, también se considera que las enfermedades sistémicas generales (diabetes, osteoporosis, etc.) pueden afectar la esperanza de vida de estos tratamientos. ${ }^{1}$ Las enfermedades periimplantarias que se pueden desarrollar incluyen la mucositis periimplantaria y la perimplantitis, siendo que la mucositis periimplantaria se define como la presencia de inflamación en la mucosa sin signos de pérdida del hueso de soporte y la periimplantitis se asocia con inflamación de la mucosa y pérdida del hueso de soporte ${ }^{2}$ informándose que hasta un $50 \%$ de los implantes dentales pueden verse afectados por la periimplantitis. ${ }^{3}$

Se ha considerado que la hiperglucemia podría ser un factor potencialmente importante en el desarrollo de complicaciones biológicas de los implantes dentales, especialmente en casos de mayor gravedad, como se observa en la diabetes mal controlada. ${ }^{4}$ Por otro lado, la evidencia actual apunta hacia un mayor riesgo de desarrollo de periimplantitis en condiciones de diabetes mellitus, lo que representa una gran preocupación en la implantología, ya que las condiciones de hiperglucemia están asociadas con la pérdida del soporte óseo.

En este sentido, el objetivo de esta revisión es la de argumentar qué evidencia científica existe acerca de la diabetes mellitus para que se considere como un factor de riesgo importante para el desarrollo de la periimplantitis.

\section{Factores de riesgo}

Los factores de riesgo son aquellas condiciones que están relacionadas con el desarrollo de la enfermedad periimplantaria, y según lo definido por Genco et al. (citado por Renvert y Quirynen) un factor de riesgo viene a ser "un factor ambiental, conductual o biológico que, si está presente, aumenta directamente la probabilidad de que ocurra una enfermedad y si está ausente o se elimina, reduce esa probabilidad". ${ }^{4}$

Considerándose que en el campo de la implantología estos factores de riesgo se pueden agrupar en tres categorías: a) factores de riesgo locales, estos son factores que pueden influir en la composición y la carga bacteriana que afectan a los implantes; como las superficies rugosas que tenga el implante, difícil acceso para realizar una adecuada higiene bucal, las bolsas periodontales remanentes que no han sido adecuadamente tratadas, bolsas periimplantarias profundas, prótesis cementadas, presencia de facetas de desgaste en coronas unitarias, rehabilitaciones de boca completa y la ausencia de tejido queratinizado adyacente a la zona del implante. ${ }^{5-7}$ b) Factores de riesgo sistémicos, que van a ser aquellos factores relacionados con el paciente y las condiciones que pueden influir a que el paciente presente mayor susceptibilidad a que desarrolle una infección, destacándose los antecedentes de enfermedad periodontal previa a la instalación del implante, la predisposición o el factor genético, el hábito al tabaco, el estado de salud general del individuo y las enfermedades sistémicas como la diabetes mellitus (diagnosticada en el momento de la instalación del implante) ${ }^{8-10} \mathrm{c}$ ) Factores relacionados con la cirugía, incluido el uso de injerto óseo, colocación inmediata, preparación y carga del sitio del implante, la distancia que existe entre implantes, la presencia de tejido mucoso delgado y profundidad de sondaje de tejidos blandos. ${ }^{11}$

\section{Periimplantitis}

La palabra periimplantitis se utiliza para describir patologías infecciosas destructivas en los tejidos blandos alrededor de los implantes dentales que provocan la pérdida ósea, ${ }^{12}$ convirtiéndose en un 
proceso destructivo que se inicia con la placa bacteriana y será mediado por la respuesta del huésped que está influenciado por factores locales, sistémicos y ambientales modificables y no modificables, con degradación de la matriz y descomposición del hueso. ${ }^{13}$

Clínicamente, la periimplantitis es definida por la presencia de enrojecimiento e inflamación de la mucosa, sangrado y/o supuración al sondaje, profundización de las bolsas adyacentes a los implantes dentales y pérdida del hueso de soporte del implante. Se ha informado que la periimplantitis ocurre en el rango de $1.4 \%$ a $53.5 \% ;^{4}$ de forma similar Dalago et al. ${ }^{12}$ señalan que en su estudio la prevalencia fue del $16.4 \%$ y del $7.3 \%$ para pacientes e implantes, respectivamente.

Sin embargo, el consenso de la Academia Europea de Osteointegración (EAO) de 2012 calculó que la prevalencia de periimplantitis es del $10 \%$ de los implantes y del $20 \%$ de los pacientes, de 5 a 10 años después de la colocación del implante. No obstante, la variación de la prevalencia surge debido a los distintos diseños de implantes y la variabilidad en los umbrales aplicados para la pérdida ósea y el análisis de tejidos blandos. ${ }^{11}$

Se supone que la mucositis periimplantaria precede a la periimplantitis, en tal razón las características histopatológicas de las lesiones periimplantarias se han evaluado en comparación con la mucositis periimplantaria, donde las lesiones de periimplantitis albergaban más granulocitos neutrófilos y mayores proporciones de células B (CD19+); y hay un dominio de células plasmáticas y linfocitos que se caracterizaron por proporciones más grandes de leucocitos polimorfonucleares y macrófagos, así como mayores proporciones de células plasmáticas, macrófagos y neutrófilos, mayor densidad de estructuras vasculares externas y laterales al infiltrado celular. ${ }^{14}$

Microbiológicamente, la periimplantitis se asocia con recuentos más altos de 19 especies bacterianas, incluidas Porphyromonas gingivalis y Tannerella forsythia; mayor frecuencia de patógenos oportunistas (Pseudomonas aeruginosa y Staphylococcus aureus), y organismos fúngicos (Candida albicans, Candida boidinii, Penicillium spp., Rhodotorula laryngis, Paecilomyces spp.), y también virus (citomegalovirus humano, virus de Epstein-Barr); definiéndose así, como una infección bastante compleja y heterogénea. ${ }^{14-16}$

\section{La diabetes mellitus como factor de riesgo de la periimplantitis}

La diabetes mellitus (DM) es un trastorno metabólico crónico que conduce a la hiperglucemia, lo que plantea múltiples complicaciones provocadas por micro y macroangiopatía. Comprende un grupo de enfermedades metabólicas donde el tipo 1 describe una destrucción autoinmune de las células $\beta$, productoras de insulina, y el tipo 2 , que es el que produce el glucagón y se caracteriza por la resistencia a la insulina: ${ }^{17}$ en tal razón, es importante saber qué tipo de diabetes padece el paciente, si existe alguna terapia, qué tipo de terapia, el grado de control glucémico y la duración de la enfermedad, para que se pueda determinar las complicaciones y los efectos secundarios derivados de la diabetes. ${ }^{18}$ La prevalencia global de diabetes en la población adulta se estima en alrededor del $8 \%$, estimándose que en el año 2017 aproximadamente 450 millones de adultos se vieron afectados por DM y se espera que esta prevalencia aumente a 693 millones para el 2045 en todo el mundo. ${ }^{19}$

La diabetes tipo 2 (DM2) es la principal forma de diabetes que afecta al 90-95 \% de la población que sufre de diabetes, convirtiéndose en un factor con un $50 \%$ más de riesgo de periimplantitis, planteando que la gravedad de la periimplantitis aumenta con un peor control glucémico en la DM2. ${ }^{18}$

En el pasado, la diabetes se consideró durante mucho tiempo como un factor de riesgo relativo 
para los implantes dentales. Por el contrario, hoy hay una evidencia indirecta de pacientes con diabetes que se benefician de la rehabilitación oral basada en la terapia con implantes dentales, ya que una adecuada rehabilitación dental permite al paciente mejorar la nutrición y el control metabólico. ${ }^{17}$

Las alteraciones inmuno-histoquímicas, como el deterioro de la vascularización y la angiogénesis inherente a un microambiente de DM2, afectan la curación ósea al retrasar la cicatrización de las heridas; tiende a reducir la neoformación de hueso y deteriora el proceso de osteogénesis. ${ }^{20} \mathrm{~A}$ la par, va a alterar las respuestas inmunitarias del hospedero, produciendo que esta respuesta aumente hacia el dominio proinflamatorio, provocando una sobreproducción de múltiples citocinas proinflamatorias en respuesta a la biopelícula periimplantaria como IL-1, IL-6, IL-8, TNF-alfa, receptores de quimiocinas CCR5 y CXR3; donde la gravedad de la desregulación empeora con un mal control de la diabetes. ${ }^{21,22}$ La modulación inmunitaria desfavorable es un mecanismo que puede ser causa de la mayor susceptibilidad a la periimplantitis que se observa en los diabéticos. Otros mecanismos por los que la periimplantitis se agrava en un paciente diabético mal controlado incluyen posibles alteraciones en la composición de la placa bacteriana y la acumulación de productos finales de glicación avanzada. ${ }^{13,23}$

Varios autores han indicado que los pacientes con diabetes tienen un mayor riesgo de periimplantitis, tal como Monje et al. ${ }^{13}$ consideran en su metaanálisis que la diabetes mellitus se asocia a un mayor riesgo de periimplantitis, independientemente del hábito del tabaco, pero no a la mucositis periimplantaria.

Alqahtani et al. ${ }^{24}$ evaluaron clínicamente las variables inflamatorias periimplantarias, indicando que estas fueron peores en fumadores y no fumadores con DM2 que no fumadores sin DM2, por lo que el estado de hiperglucemia crónica parece ser un mediador más fuerte de la inflamación periimplantaria que el tabaquismo en pacientes con DM2.

Alrabiah et al. ${ }^{25}$ hallaron que los parámetros clínicos y radiográficos periimplantarios fueron peores y los niveles de productos finales de glicación avanzada (AGE) en los niveles del líquido sulcular periimplantario (PISF) aumentaron en individuos con prediabetes y DM2; resaltando la importancia que tienen los AGE al jugar un papel importante en la inflamación periimplantaria y el desarrollo de la periimplantitis en la prediabetes y la DM2. De manera similar, Al-Askar et al. ${ }^{22}$ describen que en los pacientes con diabetes la gravedad de los parámetros de índice de placa periimplantaria, sangrado al sondaje, la profundidad de sondaje, la pérdida de hueso marginal y los niveles de IL-1 $\beta$ e IL-6 en saliva total; parecen estar influenciadas más por el estado glucémico.

En este mismo sentido, Gómez-Moreno et al. ${ }^{26}$ observaron una mayor pérdida de hueso alveolar entre los pacientes diabéticos, lo que sugiere que hay una relación proporcional a los niveles de glucemia, observando una mayor pérdida de hueso marginal en niveles más altos de HbA1c; esto puede explicarse por una mayor producción de citocinas proinflamatorias (interleucina [IL] -1B y -6 y factor de necrosis tumoral alfa [TNF-a]) en el suero y el líquido crevicular gingival debido a la interacción acelerada de los productos de glicación en pacientes diabéticos.

Al-Sowygh et al. ${ }^{27}$ informaron que el estado de los implantes dentales en pacientes con alto nivel glucémico mostró resultados comprometidos con respecto a la profundidad de sondaje y la pérdida de hueso marginal en comparación con individuos sistémicamente sanos, siendo recomendable controlar los niveles de hemoglobina glicosilada $(\mathrm{HbA} 1 \mathrm{c})$ para evitar daños periimplantarios.

Papi et al. $^{28}$, en su metaanálisis, informaron que hay un $50 \%$ más de riesgo de detectar periimplantitis en sujetos con diabetes/hiperglucemia en comparación con los no diabéticos.

De forma curiosa, varios estudios no lograron identificar la diabetes como un riesgo para la periimplantitis, como en el estudio de French et al. ${ }^{11}$ no encontraron que la diabetes (tipo 1 y tipo 2 ) tuviera un efecto significativo sobre la medida de la pérdida ósea; sin embargo, la mayoría eran de 
tipo 2 y el seguimiento promedio fue de $<4$ años, por lo que algunos casos de diabetes aún pueden sucumbir a más medida de la pérdida ósea, por lo que sugieren que se necesitan más pacientes y un periodo de seguimiento más prolongado para evaluar mejor este resultado.

Meza et al. ${ }^{19}$ dan a conocer que la evidencia real indica altos niveles de supervivencia y bajos niveles de falla de los implantes insertados en pacientes con DM; sin embargo, la DM se evaluó como un todo en la mayoría de los estudios y la influencia real de la hiperglucemia en el fracaso de los implantes aún es incierta; además, la DM sí parece estar asociada a un alto riesgo de periimplantitis.

Khandelwal et al ${ }^{29}$, en su estudio, solo incluyeron pacientes con DM mal controlada, y a pesar de que observaron algunas complicaciones (inflamación de los tejidos blandos, rotación del implante, dolor durante la activación del cicatrizal) lograron alcanzar un $98 \%$ de éxito del total de los 24 pacientes con DM2 estudiados. Algo similar ocurre en el estudio de Eskow et al., ${ }^{30}$ donde las tasas de supervivencia fueron del $98.6 \%$ después de 1 año y del $96.6 \%$ a los 2 años, y la complicación más común fue la mucositis periimplantaria con un $29 \%$; respaldando la aplicación de implantes en personas con DM2 con un control glucémico deficiente.

Finalmente, Naujokat et al. ${ }^{17}$ sostienen que los implantes dentales son procedimientos seguros y predecibles para la rehabilitación dental en diabéticos; con una supervivencia similar a la de pacientes sanos dentro de los primeros 6 años, pero a largo plazo, de hasta 20 años, hay una reducción de la supervivencia de los implantes en pacientes diabéticos; siendo necesaria una terapia de apoyo de antibióticos profilácticos y enjuague bucal con clorhexidina para reducir las complicaciones. Del mismo modo Chambrone y Palma ${ }^{31}$ argumentan que no hay información definitiva sobre el desarrollo y la pérdida ósea periimplantaria asociada con la DM2; de ahí que estos pacientes no controlados presentan peores resultados; sin embargo, los implantes dentales pueden ser exitosos cuando los niveles glucémicos y la higiene bucal se mantienen estrictamente.

\section{Conclusión}

Se concluye, en base a esta revisión, que, a pesar que no existe una evidencia concluyente, es dable considerar que existe relación entre la presencia de la diabetes mellitus y la periimplantitis, en tal razón se debe considerar a esta enfermedad como un factor de riesgo, siendo aconsejable que el paciente diabético mantenga siempre controlado su nivel de glucosa, ya que existe un mayor riesgo de patología periimplaria en pacientes con diabetes mellitus.

\section{Referencias}

1. Oates TW, Huynh-Ba G, Vargas A, Alexander P, Feine J. A critical review of diabetes, glycemic control, and dental implant therapy. Clin Oral Implants Res. 2013 Feb;24(2):117-27. doi: 10.1111/j.1600-0501.2011.02374.x.

2. Berglundh T, Armitage G, Araujo MG, Avila-Ortiz G, Blanco J, Camargo PM, et al. Peri-implant diseases and conditions: Consensus report of workgroup 4 of the 2017 World Workshop on the Classification of Periodontal and Peri-Implant Diseases and Conditions. J Clin Periodontol. 2018 Jun;45 Suppl 20:S286-S291. doi: 10.1111/jcpe.12957.

3. de Oliveira PGFP, Bonfante EA, Bergamo ETP, de Souza SLS, Riella L, Torroni A, Benalcazar Jalkh EB, Witek L, Lopez CD, Zambuzzi WF, Coelho PG. Obesity/Metabolic Syndrome and Diabetes Mellitus on Peri-implantitis. Trends Endocrinol Metab. 2020Aug;31(8):596-610. doi: 10.1016/j.tem.2020.05.005.

4. Renvert S, Quirynen M. Risk indicators for peri-implantitis. A narrative review. Clin Oral 
Implants Res. 2015 Sep;26 Suppl 11:15-44. doi: 10.1111/clr.12636.

5. Linkevicius T, Puisys A, Vindasiute E, Linkeviciene L, Apse P. Does residual cement around implant-supported restorations cause peri-implant disease? A retrospective case analysis. Clin Oral Implants Res. 2013 Nov;24(11):1179-84. doi: 10.1111/j.1600-0501.2012.02570.x.

6. Mawhinney J, Connolly E, Claffey N, Moran G, Polyzois I. An in vivo comparison of internal bacterial colonization in two dental implant systems: identification of a pathogenic reservoir. Acta Odontol Scand. 2015 Apr;73(3):188-94. doi: 10.3109/00016357.2014.978365.

7. Lin GH, Chan HL, Wang HL. The significance of keratinized mucosa on implant health: a systematic review. J Periodontol. 2013 Dec;84(12):1755-67. doi: 10.1902/jop.2013.120688.

8. Daubert DM, Weinstein BF, Bordin S, Leroux BG, Flemming TF. Prevalence and predictive factors for peri-implant disease and implant failure: a cross-sectional analysis. J Periodontol. 2015 Mar;86(3):337-47. doi: 10.1902/jop.2014.140438.

9. Roccuzzo M, Bonino L, Dalmasso P, Aglietta M. Long-term results of a three arms prospective cohort study on implants in periodontally compromised patients: 10-year data around sandblasted and acid-etched (SLA) surface. Clin Oral Implants Res. 2014 Oct;25(10):110512. doi: $10.1111 / \mathrm{clr} .12227$.

10. Renvert S, Polyzois I. Risk indicators for peri-implant mucositis: a systematic literature review. J Clin Periodontol. 2015Apr;42 Suppl 16:S172-86. doi: 10.1111/jcpe.12346.

11. French D, Grandin HM, Ofec R. Retrospective cohort study of 4,591 dental implants: Analysis of risk indicators for bone loss and prevalence of peri-implant mucositis and peri-implantitis. J Periodontol. 2019 Jul;90(7):691-700. doi: 10.1002/JPER.18-0236.

12. Dalago HR, Schuldt Filho G, Rodrigues MA, Renvert S, Bianchini MA. Risk indicators for Periimplantitis. A cross-sectional study with 916 implants. Clin Oral Implants Res. 2017 Feb;28(2):144-150. doi: 10.1111/clr.12772.

13. Monje A, Catena A, Borgnakke WS. Association between diabetes mellitus/hyperglycaemia and peri-implant diseases: Systematic review and meta-analysis. J Clin Periodontol. 2017 Jun;44(6):636-648. doi: 10.1111/jcpe.12724.

14. Schwarz F, Derks J, Monje A, Wang HL. Peri-implantitis. J Periodontol. 2018 Jun;89 Suppl 1:S267-S290. doi: 10.1002/JPER.16-0350.

15. Zhuang LF, Watt RM, Mattheos N, Si MS, Lai HC, Lang NP. Periodontal and peri-implant microbiota in patients with healthy and inflamed periodontal and peri-implant tissues. Clin Oral Implants Res. 2016 Jan;27(1):13-21. doi: 10.1111/clr.12508.

16. Yan X, Lu H, Zhang L, Zhu B, Piao M, Huang B, Zhang H, Meng H. A three-year study on periodontal microorganisms of short locking-taper implants and adjacent teeth in patients with history of periodontitis. J Dent. 2020 Apr;95:103299. doi: 10.1016/j.jdent.2020.103299.

17. Naujokat $\mathrm{H}$, Kunzendorf $\mathrm{B}$, Wiltfang J. Dental implants and diabetes mellitus-a systematic review. Int J Implant Dent. 2016 Dec;2(1):5. doi: 10.1186/s40729-016-0038-2.

18. Yu T, Acharya A, Mattheos N, Li S, Ziebolz D, Schmalz G, Haak R, Schmidt J, Sun Y. Molecular mechanisms linking peri-implantitis and type 2 diabetes mellitus revealed by transcriptomic analysis. PeerJ. 2019 Jun 21;7:e7124. doi: 10.7717/peerj.7124.

19. Meza Maurício J, Miranda TS, Almeida ML, Silva HD, Figueiredo LC, Duarte PM. An umbrella review on the effects of diabetes on implant failure and peri-implant diseases. Braz Oral Res. 2019 Sep 30;33(suppl 1):e070. doi: 10.1590/1807-3107bor-2019.vol33.0070.

20. Marin C, Luyten FP, Van der Schueren B, Kerckhofs G, Vandamme K. The Impact of Type 2 Diabetes on Bone Fracture Healing. Front Endocrinol (Lausanne). 2018 Jan 24;9:6. doi: 
10.3389/fendo.2018.00006.

21. Nielsen TB, Pantapalangkoor P, Yan J, Luna BM, Dekitani K, Bruhn K, Tan B, Junus J, Bonomo RA, Schmidt AM, Everson M, Duncanson F, Doherty TM, Lin L, Spellberg B. Diabetes Exacerbates Infection via Hyperinflammation by Signaling through TLR4 and RAGE. mBio. 2017 Aug 22;8(4):e00818-17. doi: 10.1128/mBio.00818-17.

22. Al-Askar M, Ajlan S, Alomar N, Al-Daghri NM. Clinical and Radiographic Peri-Implant Parameters and Whole Salivary Interleukin-1 $\beta$ and Interleukin-6 Levels among Type-2 Diabetic and Nondiabetic Patients with and without Peri-Implantitis. Med Princ Pract. 2018;27(2):133-138. doi: 10.1159/000488032.

23. Gulia S, Bhatt V, Shetty M, Prasad KD, Gupta P. Effect of Type II Diabetes Mellitus, Candida Albicans and Streptococcus Mutans on the Biofilm Formation on Prosthetic Materials. J Contemp Dent Pract. 2018 Dec 1;19(12):1538-1545.

24. Alqahtani F, Alqhtani N, Alkhtani F, Devang Divakar D, Al-Kheraif AA, Javed F. Clinicoradiographic markers of peri-implantitis in cigarette-smokers and never-smokers with type 2 diabetes mellitus at 7-years follow-up. J Periodontol. 2020 Sep;91(9):1132-1138. doi: 10.1002/JPER.19-0501.

25. Alrabiah M, Al-Aali KA, Al-Sowygh ZH, Binmahfooz AM, Mokeem SA, Abduljabbar T. Association of advanced glycation end products with peri-implant inflammation in prediabetes and type 2 diabetes mellitus patients. Clin Implant Dent Relat Res. 2018 Aug;20(4):535-540. doi: 10.1111/cid.12607.

26. Gómez-Moreno G, Aguilar-Salvatierra A, Rubio Roldán J, Guardia J, Gargallo J, CalvoGuirado JL. Peri-implant evaluation in type 2 diabetes mellitus patients: a 3-year study. Clin Oral Implants Res. 2015 Sep;26(9):1031-5. doi: 10.1111/clr.12391.

27. Al-Sowygh ZH, Ghani SMA, Sergis K, Vohra F, Akram Z. Peri-implant conditions and levels of advanced glycation end products among patients with different glycemic control. Clin Implant Dent Relat Res. 2018 Jun;20(3):345-351. doi: 10.1111/cid.12584.

28. Papi P, Letizia C, Pilloni A, Petramala L, Saracino V, Rosella D, Pompa G. Peri-implant diseases and metabolic syndrome components: a systematic review. Eur Rev Med Pharmacol Sci. 2018 Feb;22(4):866-875. doi: 10.26355/eurrev_201802_14364.

29. Khandelwal N, Oates TW, Vargas A, Alexander PP, Schoolfield JD, Alex McMahan C. Conventional SLA and chemically modified SLA implants in patients with poorly controlled type 2 diabetes mellitus--a randomized controlled trial. Clin Oral Implants Res. 2013 Jan;24(1):13-9. doi: 10.1111/j.1600-0501.2011.02369.x.

30. Eskow CC, Oates TW. Dental Implant Survival and Complication Rate over 2 Years for Individuals with Poorly Controlled Type 2 Diabetes Mellitus. Clin Implant Dent Relat Res. 2017 Jun;19(3):423-431. doi: 10.1111/cid.12465.

31. Chambrone L, Palma LF. Current status of dental implants survival and peri-implant bone loss in patients with uncontrolled type-2 diabetes mellitus. Curr Opin Endocrinol Diabetes Obes. 2019Aug;26(4):219-222. doi: 10.1097/MED.0000000000000482. 